\title{
Observation of Compositional Heterogeneity in Poly(styrene sulfonate) Using Frontal Analysis Continuous Capillary Electrophoresis
}

\author{
Bin Zhang, ${ }^{\dagger}$ Toshiaki Hattori, ,t, and Paul L. Dubin*,t \\ Department of Chemistry, Indiana University-Purdue University at Indianapolis, \\ Indianapolis, Indiana 46202-3274, and Research Center for Chemometrics, \\ Toyohashi University of Technology, Toyohashi, J apan 441-8580 \\ Received J anuary 9, 2001; Revised Manuscript Received J une 26, 2001
}

\begin{abstract}
Sulfonated polystyrene is frequently studied as a model polyelectrolyte because of its narrow molecular weight distribution, corresponding to that of the precursor, anionically polymerized polystyrene. Frontal analysis continuous capillary electrophoresis (FACCE) was carried out for polystyrenesulfonate (PSS) samples from various commercial sources. Whereas some of the electropherograms appeared as abrupt and discontinuous, indicating a single uniform mobility, others were sigmoi dal, corresponding to a distribution of mobilities. This distribution must reflect sample heterogeneity, possibly due in part to the molecular weight distribution (MWD) for the low-MW samples, but only attributable to chemical heterogeneity for the higher-MW samples. Size-exclusion chromatography (SEC) was used to establish the possibility of some MWD contribution to the distribution of mobilities for the low-MW PSSs, but not for the high-MW ones. Because a high-MW sample with a broad MWD prepared by polymerization of styrenesulfonate did not show a mobility distribution, the only plausible explanation for those distributions is compositional heterogeneity, i.e., chain-to-chain variations in the degree of sulfonation.
\end{abstract}

\section{Introduction}

Poly(styrenesulfonate) (PSS) has been frequently considered a model linear polyelectrolyte, ${ }^{1-4}$ and indeed, many of its properties are typical of ionic polymers. Various workers have considered the properties of PSS to embody typical polyelectrolyte behavior, including the Mark-Houwink exponent and its ionic strength dependence, ${ }^{5}$ the dependence of the reduced viscosity on concentration and shear rate, ${ }^{4}$ and the effect of those two variables on the mutual diffusion coefficient from dynamic light scattering. ${ }^{6}$ On the other hand, several observations have been made to indicate that PSS is hydrophobic. Turro and Okubo found that cationic fluorescent probes in PSS solutions show spectral shifts corresponding to a highly hydrophobic microenvironment, intermediate in value between those of micelles and n-hexane. ${ }^{7}$ They also invoked hydrophobic interactions between PSS and probes to explain the increase in excimer emission of the fluorophores. ${ }^{8} \mathrm{~K}$ wak and coworkers ${ }^{9}$ found that PSS binds cationic surfactants more strongly than sodium dextran sulfate and attributed this to the "hydrophobicity of the PSS backbone", later substantiating this conclusion by observations of NMR ring current effects. ${ }^{10}$ Last, Mori et al. ascribed the deviation of PSS size-exclusion calibration curves from those of the reference polymer pullulan to the hydrophobicity of the former. ${ }^{11}$

PSS has been widely used as a calibration standard or model polyelectrolyte because it can be prepared from narrow-distribution polystyrene, and thus is considered to be well-characterized with respect to molecular weight and molecular weight distribution. However, the sulfonation process might be problematic, resulting in sulfone formation and cross-linking at high degrees of sulfonation. Possibly for this reason, the commercial

* To whom correspondence should be addressed.

† Indiana University-Purdue University at Indianapolis.

‡ Toyohashi University of Technology. samples of PSS used in most studies, typically originating in the past from Pressure Chemical Company, often have nominal degrees of sulfonation ranging from 80 to $90 \%$. If unsulfonated groups were regularly surrounded by sulfonated residues, the properties of the polymer might be only weakly affected. However, if the sulfonation process were one in which solubility effects guaranteed that the more highly sulfonated species continued to react preferentially, then the degree of sulfonation measured by el emental analysis could represent a very broad compositional distribution; in other words, a significant fraction of the material could contain sequences of unsulfonated polystyrene that could strongly influence aggregation and other behavior. Unfortunately, most studies with PSS have failed to consider the possibility that "hydrophobic behavior" or aggregation might arise from such compositional effects.

Few techniques are available to investigate compositional heterogeneity in derivatized polymers. ${ }^{13} \mathrm{C}$ NMR spectroscopy can provide information on sequence distribution, but this arduous procedure would not reveal variability among polymer molecules, and exactly this chain-to-chain polydispersity is of special interest here. The relatively new technique of matrix-assisted laser desorption/ionization time-of-flight mass spectrometry (MALDI-TOF-MS) has been established as a powerful method for the determination of molecular weight distributions (MWD) of synthetic polymers. ${ }^{12-14} \mathrm{How}$ ever, the sensitivity of MALDI-TOF-MS to experimental parameters is well-known, and single-chain resolution is obtained only in mass spectra of samples of MW below $10000 \mathrm{Da}$.

Capillary electrophoresis (CE) provides a new approach to the characterization of charged polymers. ${ }^{15,16}$ Hoagland reported the use of CE to measure accurately the free solution mobility $\mu_{0}$ of highly charged, watersol uble polyelectrolytes as a function of ionic strength I and degree of polymerization $\mathrm{N} .{ }^{17}$ Cottet used a semiempirical approach to describe the electrophoretic mobility 
of polystyrenesulfonates of various degrees of sulfonation in free solution. ${ }^{18}$ We demonstrated the use of frontal analysis continuous capillary electrophoresis (FACCE) for characterization of copolymers of acrylamidopropylsulfonic acid (sodium salt) (NaAMPS) and acrylamide $^{19}$ and concluded that the distribution of mobilities detected by FACCE could be related to a distribution of chains with different AMPS contents. In the present work, we use the same approach to observe compositional heterogeneity in commercial samples of PSS prepared by sulfonation of polystyrene and compare the results to those for PSS prepared by polymerization of stryrenesulfonate.

\section{Experimental Section}

Materials. The commercial PSS samples used are identified according to molecular weight (MW): 1.6K, 5.4K, 35.2K, and 88K from Pressure Chemical Co. (Pittsbugh, PA); 70K and 500K from Polysciences (Warrington, PA); and 12K from Lion Co. (Tokyo). A sample with a MW of 250K was synthesized from sodium styrenesulfonate monomer in this laboratory using a method described elsewhere. ${ }^{20}$ The $70 \mathrm{~K}$ polymer was dialyzed and freeze-dried before use, but all others were used as received. Phosphate buffers were prepared from monobasic and dibasic salts of sodium phosphate obtained from Fisher Scientific Co. (Fair Lawn, NJ) and J . T. Baker Chemical Co. (Phillipsburg, NJ ). Mesityl oxide was purchased from Aldrich Chemical Co. (Milwaukee, WI). Milli-Q water was used throughout this work.

Capillary E lectrophoresis. Capillary el ectrophoresis (CE) was carried out on a P/ACE 5500 instrument (Beckman, Fullerton, CA). A bare fused-silica capillary (Restek, Bellefonte, PA) of dimensions $50 \mu \mathrm{m} \times 27 \mathrm{~cm}$ ( $20 \mathrm{~cm}$ to the detector) was used. UV detection at $200 \mathrm{~nm}$ was employed. The voltage applied across the capillary was fixed at $10 \mathrm{kV}$, and the temperature was maintained at $25.0 \pm 0.1^{\circ} \mathrm{C}$ with fluorocarbon coolant. The run buffer was $\mathrm{pH} 6.9, \mathrm{I}=0.04 \mathrm{M}$ phosphate that filtered through $0.2-\mu \mathrm{m}$ PP W/GMF filters (Whatman). The concentration of each PSS sample was about $0.2 \mathrm{~g} / \mathrm{L}$. All samples were completely soluble in run buffer except for $500 \mathrm{~K}$, which was not fully soluble and, therefore, was filtered through 0.1- $\mu \mathrm{m}$ AT filters (Whatman). For each CE experiment, a single 3-s injection of neutral marker (mesityl oxide) preceded the frontal analysis continuous capillary electrophoresis (FACCE ). Between runs, the capillary was subjected to a 3-min rinse with $0.1 \mathrm{~N} \mathrm{NaOH}$, water, and run buffer in that order. The detailed description of FACCE can be found elsewhere. ${ }^{20,21}$

The electrophor etic mobility was calculated by subtracting the sample's apparent el ectrophoretic mobility from the electroosmotic flow (EOF) of the run buffer, as shown in eq 1

$$
\mu=\frac{\mathrm{v}_{0}-\mathrm{V}_{1}}{\mathrm{E}}=\frac{\mathrm{IL}}{\mathrm{V}}\left(\frac{1}{\mathrm{t}_{\mathrm{m}}}-\frac{1}{\mathrm{t}_{\mathrm{s}}}\right)
$$

where $v_{0}$ and $v_{1}$ are the el ectroosmotic vel ocity and the solute vel ocity, respectively; $\mathrm{E}$ is the applied electric field strength; I is the effective length of the capillary, and $L$ is the total length of the capillary (both in $\mathrm{cm}$ ); $\mathrm{V}$ is the applied voltage; and $\mathrm{t}_{\mathrm{m}}$ and $t_{s}$ are the migration times of the reference marker (mesityl oxide) and the sample, respectively. Multiple runs showed that the reproducibility of $\mu$ is within $\pm 0.6 \%$.

Size-Exclusion Chromatography. Size-exclusion chromatography (SE C) was carried out on an apparatus including a Minipump (Milton Roy), a model 7010 injector (Rheodyne) equipped with a 100- $\mu \mathrm{L}$ sample loop, and an R401 differential refractometer (Waters). A Superose 12 (Pharmacia, Piscataway $\mathrm{NJ}$ ) column was eluted at a rate of about $0.45 \mathrm{~mL} / \mathrm{min}$. The aqueous mobile phase was $\mathrm{pH} 7, \mathrm{I}=0.1 \mathrm{M}$ (or $0.08 \mathrm{M}$ ) phosphate buffer. The column was calibrated using PSS MW standards.

\section{Results and Discussion}

To correct for the effect of variable el ectroosmotic flow on migration time, we converted the times to mobilities

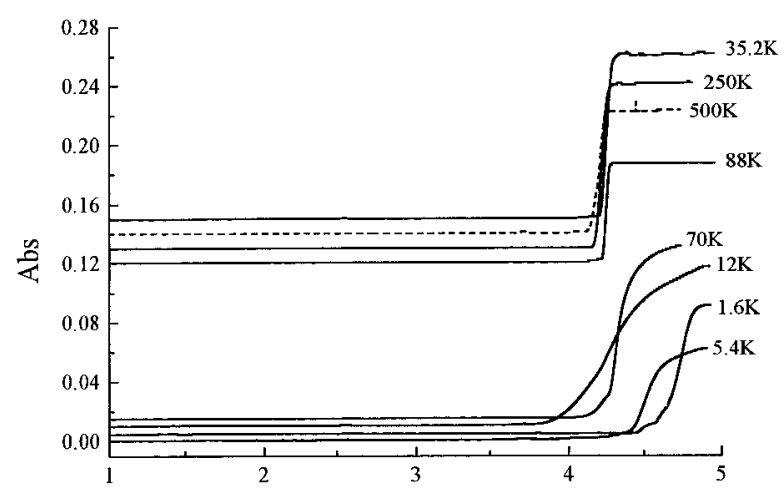

$\mu,\left(\mathrm{cm}^{2} / \mathrm{V} . \mathrm{s}\right) \times 10^{4}$

Figure 1. FACCE electropherograms of PSS (upper set shifted for clarity).

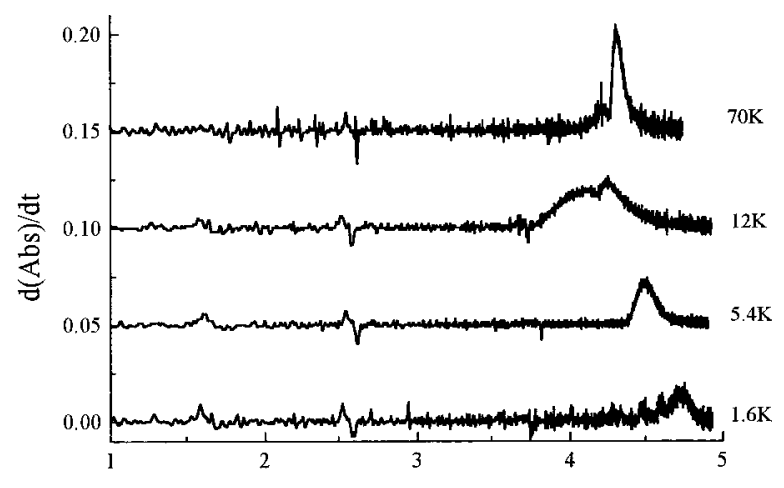

$\mu,\left(\mathrm{cm}^{2} / \mathrm{V} . \mathrm{s}\right) \times 10^{4}$

Figure 2. Mobility distribution obtained by differentiation of the electropherograms of PSSs (1.6K, 5.4K, 12K, and 70K), presented as d(Abs)/dt (arbitrary units) vs $\mu$.

by eq 1 and obtained the FACCE electropherograms in the form of Figure 1. The electropherogram for the 250K sample prepared by polymerization of NaSS is discontinuous and abrupt, with the solute eluting as a single front, as are those of the $35.2 \mathrm{~K}$ and $88 \mathrm{~K}$ samples; the electropherogram for the 500K polymer also exhibits a plateau but with a discernible preceding slope. However, the electropherograms for the $1.6 \mathrm{~K}, 5.4 \mathrm{~K}, 12 \mathrm{~K}$, and $70 \mathrm{~K}$ samples appear as sigmoidal or more complex traces. In previous work, ${ }^{19}$ we observed that copolymers of acrylamide and acrylamidomethyl propylsulfonate yiel ded FACCE electropherograms with discernible slopes, in contrast to the discontinuous and abrupt el ectropherogram for hyaluronic acid, and we related that difference to the absence of any chemical heterogeneity in the biopolymer, in contrast to the synthetic materials. A similar interpretation seems reasonable in the present case. Differentiation of the electropherograms of the PSS samples leads to the differential distributions shown in Figures 2 and 3. We note the strikingly narrow Gaussian distributions for the $250 \mathrm{~K}$, 88K , and 35.2K samples, the broad distributions for the $12 \mathrm{~K}, 5.4 \mathrm{~K}$, and $1.6 \mathrm{~K}$ samples, and the bimodal distributions for the $500 \mathrm{~K}$ and 70K samples. Cottet et al. ${ }^{18}$ also obtained peaks of varying breadth by direct injection of PSS samples with different degrees of sulfonation and even observed a bimodal distribution for one sample.

Synthetic copolymers are heterogeneous with regard to both chemical composition and degree of polymerization, $\mathrm{N}$. We now consider the effects of these two types of heterogeneities on electrophoretic behavior. In addition to interchain heterogeneity, we might imagine 


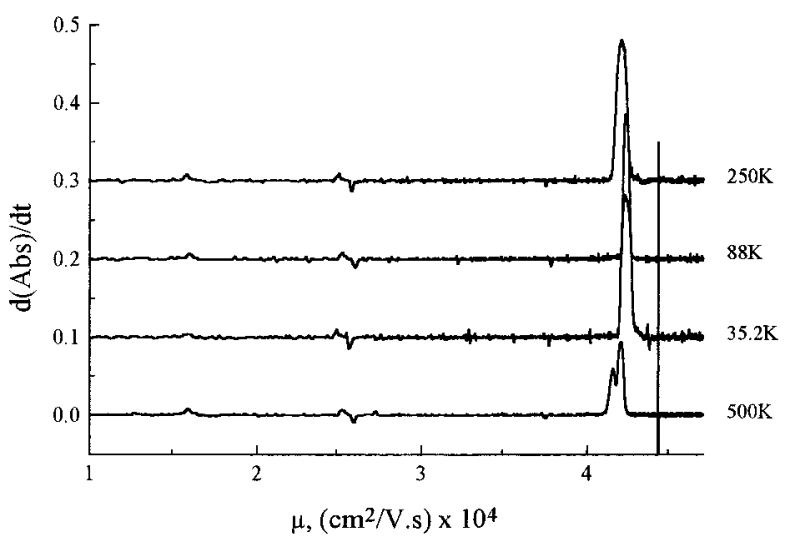

Figure 3. Mobility distribution obtained by differentiation of the electropherograms of PSSs $(35.2 \mathrm{~K}, 88 \mathrm{~K}, 250 \mathrm{~K}$, and $500 \mathrm{~K})$, presented as $\mathrm{d}(\mathrm{Abs}) / \mathrm{dt}$ (arbitrary units) vs $\mu$.

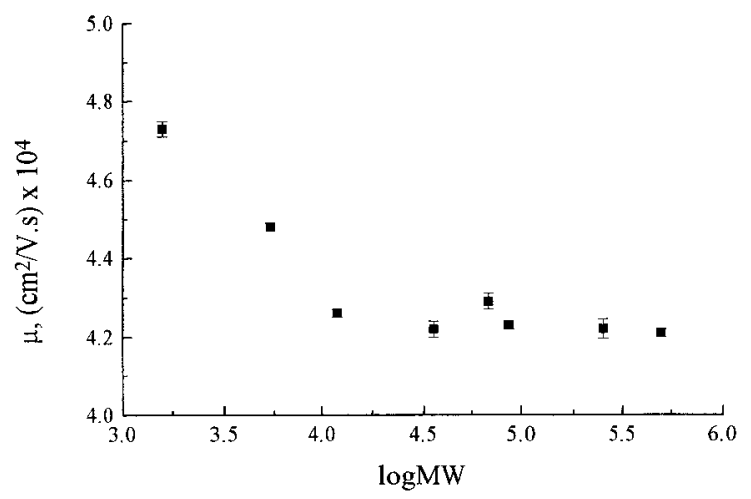

Figure 4. Dependence of mobility on molecular weight. The reproducibility of sequential runs is reflected in \pm 1.0 standard deviation error bars.

nonuniformities within each chain i.e., "positional polydispersity" and its effect on mobility. Long et al. ${ }^{22}$ considered this effect for the more extreme case of polyampholytes. In that case, a sequence of positive units can exert under an electrical field a tension on an oppositely charged sequence, leading to deformation so that the mobility can be sequence-dependent, particularly at high salt concentrations. However, in the present case of nonionic/ionic polymer at low salt concentrations, the magnitude of sequence heterogeneity ("blockiness") needed to create such an effect seems unlikely.

In general, the electrophoretic mobility of flexible linear polyelectrolytes is known to be essentially independent of MW. The results shown in Figure 4 indicate that the mean mobility (peak value in Figures 2 and 3) is independent of $\mathrm{MW}$ for $\mathrm{MW}>10^{4}$ and increases gradually by ca. $10 \%$ as the MW decreases to $1.6 \times 10^{3}$. The effect of polymer MW on mobility via the solution viscosity must be negligible, as shown by the fact that the measured mobility of the 5.4K sample is not altered by co-injection of 88K polymer. Consistent with our results, Poli et al. found 1.8K PSS has a higher mobility than $8 \mathrm{~K}-1200 \mathrm{~K}$ PSSs. ${ }^{23} \mathrm{H}$ oagland ${ }^{17}$ observed that the mobility, $\mu_{0}$, of PSS is independent of the degree of polymerization $\mathrm{N}$ for $\mathrm{N}>100$ but found more complex trends at lower $\mathrm{N}$. F or example, a sample with $\mathrm{N}=8$ produced a number of electropherogram peaks, some of them corresponding to $\mu_{0}$ greater than that of high-MW PSS. These peaks were interpreted as electrophoretic separation by $\mathrm{N}$, and it was suggested that $\mu_{0}$ passed through a maximum somewhere in the range $5<\mathrm{N}<$ 25 . Cottet similarly reported that the electrophoretic mobility of fully sulfonated PSS oligomers first increases strongly with $N$, reaches a maximum at $N \approx 10$, and then decreases sl owly to a constant value at a threshol d value $\mathrm{N}_{\mathrm{FD}}$ ( $\mathrm{N}_{\mathrm{FD}}$ depends on the ionic strength, ranging from about 100 at $20 \mathrm{mM}$ ionic strength to about 800 in pure water). ${ }^{18}$

From SEC results, we calculated polydispersities for the $5.4 \mathrm{~K}$ and $12 \mathrm{~K}$ polymers as 1.05 and 1.64 , respectively. The data in Figure 4 show that the mobility is inversely and nearly linearly dependent on the MW for $10^{3}<\mathrm{MW}<10^{4}$ but essentially MW-independent for $\mathrm{MW}>10^{4}$. From this correlation between mobility and MW, the distribution of mobility can be deduced from the SEC-generated MW distribution. In this way, we found that the mobility distribution predicted from the MW range could account for up to $70 \%$ of the observed mobility range for the $5.4 \mathrm{~K}$ sample but only $40 \%$ of the observed mobility range for the 12K sample. We therefore conclude that molecular weight distribution might, in principle, account for some of the distribution of electrophoretic mobilities for the $5.4 \mathrm{~K}$ and $12 \mathrm{~K}$ polymers but not at all for the distributions of mobilities observed for the 70K or 500K PSSs. For these high-molecularweight PSS samples, the only plausible explanation for the broadened electropherograms is therefore compositional heterogeneity, i.e., chain-to-chain variations in the degree of sulfonation. ${ }^{24}$ Although commercial samples of PSS from Pressure Chemical Co. are generally reported to have average degrees of sulfonation on the order of $73-88 \%,{ }^{4}$ these results suggest that much of the sample consists of polymer chains with lower than average degrees of sulfonation. Indeed, our observation of partial solubility for 500K PSS must indicate heterogeneity of either substitution, cross-linking, or both.

Polyelectrolytes in aqueous solution are typically modeled as charged linear chains surrounded by an atmosphere of counterions. ${ }^{25-29}$ The counterion condensation theory ${ }^{30}$ states that, when the dimensionless mean linear structural charge density $(\xi)$ of the polyion exceeds the reciprocal of the valence of the counterions $Z$, counterions will condense along the polyion contour length, effectively reducing $\xi$ to $|Z|^{-1}$ (i.e., unity in this case). Here, the dimensionless linear charge density, $\xi$, is defined in SI units as

$$
\xi=\frac{\mathrm{e}^{2}}{4 \pi \epsilon_{\mathrm{r}} \epsilon_{0} \mathrm{k}_{\mathrm{B}} T \mathrm{~b}}=\frac{\lambda_{\mathrm{B}}}{\mathrm{b}}
$$

where e is the elementary charge of a proton $(C) ; \epsilon_{r}$ is the dimensionless dielectric constant of the solvent; $\epsilon_{0}$ is the permittivity of vacuum $(\mathrm{F} / \mathrm{m})$; $\mathrm{k}_{\mathrm{B}}$ is the Boltzmann constant $(\mathrm{J} / \mathrm{K})$; $\mathrm{T}$ is the temperature $(\mathrm{K})$; $b$ is the average charge spacing along the polyion chain $(\mathrm{m})$; and $\lambda_{\mathrm{B}}$ is the Bjerrum length, which corresponds to the equality of the thermal and el ectrostatic energy of two elementary charges in a medium of relative permittivity $4 \pi \epsilon_{0} \epsilon^{31}$ Polyelectrolyte mobility has been observed to rise linearly with charge density when this density is low and to show a discontinuity near $\xi=1$, as predicted by Manning theory. For a highly sulfonated poly(styrene) chain in water (neutral $\mathrm{pH}$ ), $\xi$ is about 2.53.0, so it might be thought that the dimensionless effective linear charge density is independent of the actual degree of sulfonation. ${ }^{32}$ However, the effect of ionic content on the mobility of acrylic acid/acrylamide copolymer observed by Hoagland et al. ${ }^{33}$ and for acrylamideo-methyl propanesul fonate/acrylamide copolymer 


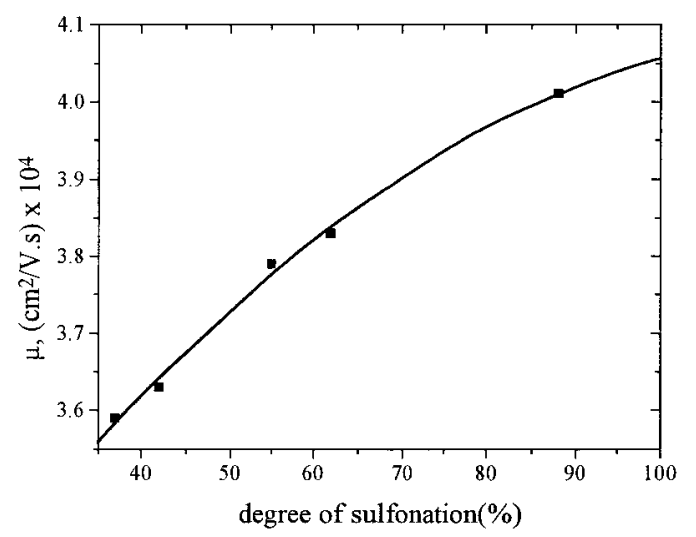

Figure 5. Dependence of mobility on degree of sulfonation for partially sulfonated PSS in I $=0.04 \mathrm{M}$ buffer; obtained by interpolation of data from ref 18 over the ionic strength range $0.00015-0.075 \mathrm{M}$

found by Dubin et al. ${ }^{34}$ both indicate that the mobility displays a modest increase with polymer charge content even beyond $\xi=1$, an observation that might be attributed to the difference between real copolymer sequence distributions and the ideal continuous line charge parameter of Manning. In any event, it is this weak dependence of mobility on charge content that makes it possible to observe the el ectrophoretic peak dispersions for several samples. From Cottet's paper, 18 we can estimate the relationship between mobility and degree of sulfonation for PSS at I $=0.04 \mathrm{M}$, as shown in Figure 5 , and we find that a $1 \%$ change in mobility corresponds to a ca. $10 \%$ change in degree of sulfonation in the range of $80-100 \%$ sulfonation. Therefore, the rather modest ranges of mobility observed for these samples must correspond to a large degree of compositional heterogeneity.

The presence of lowly sulfonated NaPSS in commercial samples could lead to aggregation effects, which could strongly affect many of the scattering and hydrodynamic measurements reported for these materials. Hydrophobic interactions among sequences of unsulfonated styrene residues might explain anomalous microscopically observable aggregates. ${ }^{35}$ They could also account in part for the fact that the effect of sulfonate content on small-angle X-ray scattering for partially sulfonated NaPSS is very different from that seen for AMPS/acrylamide copolymers. ${ }^{36}$

For high-MW polymers, the possible sequence distributions are virtually numberless, and the electropherograms are therefore continuous. For PSS oligomers, however, the possible sequences for, e.g., a decamer with an average of $90 \%$ sulfonation are finite. Thus, the possibility arises of observing discrete species in the case of low-MW PSS. Close inspection of the electropherogram of the 1.6K sample (i.e., octamer) in Figure 2 suggests such a possible pattern, and Hoagland has observed such multiple peaks for PSS $(\mathrm{N}=8) .{ }^{17}$ The presence of discrete oligomeric species is even more evident in the SEC chromatogram of Figure 6. The strong sharp peak, present in all undialyzed samples regardless of MW, is salt and marks the column void volume, $V_{0}$. At least seven later-eluting peaks can be discerned, and their retention beyond $\mathrm{V}_{0}$ eliminates the possibility that they are fully sulfonated oligomers. Because Superose bears a weak negative charge at $\mathrm{pH}$ $=7\left(\right.$ ca. $\left.8 \times 10^{-3} \mathrm{C} / \mathrm{m}^{2}\right),{ }^{37}$ fully charged NaPSS oligomer anions should, if anything, be repelled and elute early.

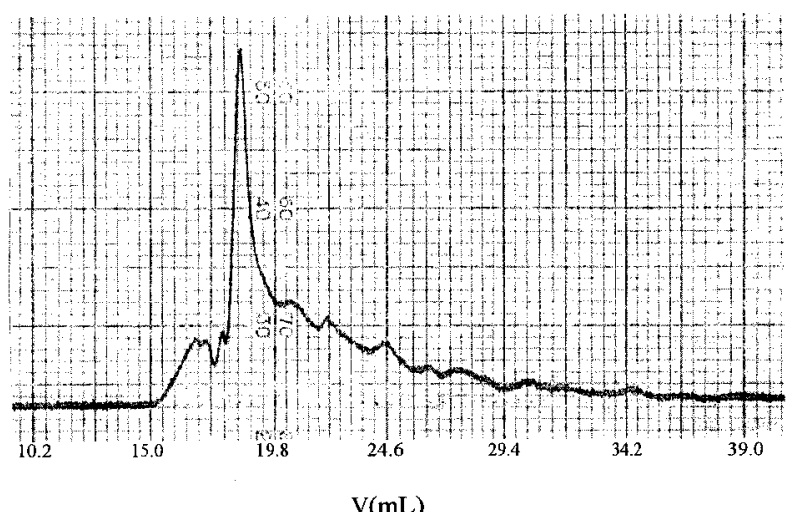

Figure 6. Size-exclusion chromatograph of 1.6K PSS (Superose $12, \mathrm{pH} \mathrm{7,} \mathrm{I=0.08} \mathrm{M} \mathrm{phosphate} \mathrm{buffer),} \mathrm{as} \mathrm{refractive} \mathrm{index}$ detector response (arbitrary units) vs elution volume.

On the other hand, the ability of Superose to retain amphiphilic solutes via hydrophobic interactions has been established by observing that the retention of linear al cohols $\mathrm{C}_{n} \mathrm{H}_{2 n+1} \mathrm{OH}$ increases systematically with $\mathrm{n}$ beyond $\mathrm{V}_{0}{ }^{38}$ These late-eluting peaks in Figure 6 can thus be identified with solutes of increasing hydrophobicity, i.e., oligomers with incomplete sulfonation.

\section{Conclusion}

The broadening of FACCE electropherograms for samples of PSS prepared by sulfonation of polystyrene must correspond to compositional heterogeneity in which the polymer molecules have a significant range of ionic contents, presumably arising from nonuniform incomplete sulfonation. Thus, considerable caution seems appropriate if PSS prepared in this manner is to be considered as a paradigm for polyelectrolyte behavior.

Acknowledgment. Support from the National Science Foundation (CHE 9987891) is acknowledged.

\section{References and Notes}

(1) Molyneux, P. Water SolubleSynthetic Polymers; CRC Press: Boca Raton, FL, 1984; Vol. II.

(2) Koene, R. S.; Mandel, M. Macromol ecules 1983, 16, 220.

(3) Wang, L.; Yu, H. Macromolecules 1988, 21, 3498.

(4) Yamanaka, J.; Matsuoka, H.; Kitano, H.; Hasegawa, M.; Ise, N. J . Am. Chem. Soc. 1990, 112, 587.

(5) Takahashi, A.; Kato, T.; Nagasawa, M.J . Phys. Chem. 1967 71, 2001.

(6) Koene, R. S.; Nicolai, T.; Mandel, M. Macromolecules 1983 $16,227$.

(7) Turro, N. J .; Okubo, T. J . Am. Chem. Soc. 1982, 104, 2985.

(8) Turro, N.; Okubo, T.; Chung, C.-J .; Emert, J .; Catena, R. J . Am. Chem. Soc. 1982, 104, 4799.

(9) Hayakawa, K.; Kwak, J. C. T.J . Phys. Chem. 1982, 86, 3866.

(10) Gao, Z.; Kwak, J. C. T. J . Colloid Interface Sci. 1988, 126, 371.

(11) Mori, S. Anal. Chem. 1989, 61, 530

(12) Raeder, H. J .; Spickermann, J .; Muellen, K. Macromol. Chem. Phys. 1995, 196, 3967

(13) Danis, P. O.; Karr, D. E. Macromolecules 1995, 28, 8548.

(14) Arakawa, R.; Watanabe, S.; Fukuo, T. Rapid Commun. Mass Spectrom. 1999, 13, 1059.

(15) Cottet, H.; Gareil, P. J . Chromatogr. A 1997, 772, 369.

(16) Grosche, O.; Bohrisch, J .; Wendler, U.; J aeger, W.; Engelhardt, H. J . Chromatogr. A 2000, 894, 105.

(17) Hoagland, D. A.; Arvanitidou, E.; Welch, C. Macromolecules 1999, 32, 6180.

(18) Cottet, H.; Gareil, P.; Theodoly, O.; Williams, C. E. Electrophoresis 2000, 21, 3529.

(19) Staggemeir, B.; Huang, Q. R.; Dubin, P. L. Anal. Chem. 2000, $72,255$.

(20) Hallberg, R. K.; Dubin, P. L. J . Phys. Chem. B 1998, 102, 8629. 
(21) Gao, J . Y.; Dubin, P. L.; Muhoberac, B. Anal. Chem. 1997, 69, 2945.

(22) Long, D.; Dobrynin, A. V.; Rubinstein, M.; Ajdari, A. J . Chem. Phys. 1998, 108, 1234.

(23) Poli, J . B.; Schure, M. R. Anal. Chem. 1992, 64, 896.

(24) Elemental analysis gave $C / S$ ratios in the range of $3.3( \pm 0.3)$ corresponding to reasonable degrees of sulfonation of about $90 \%$ for most of the samples; but excessively large C/S values (5.0 and 6.1) were found for the $12 \mathrm{~K}$ and 88K PSSs. Combined values for $\mathrm{C}, \mathrm{S}$, and $\mathrm{H}$ were not consistent with any structure. We attributed these anomalous results to incomplete combustion.

(25) Manning, G. S. Acc. Chem. Res. 1979, 12, 443.

(26) Bret, M. L.; Zimm, B. H. Biopolymers 1984, 23, 287.

(27) Naghizadeh, J. Cell Biophys. 1987, 11, 103.

(28) Manning, G. S. J . Phys. Chem. 1981, 85, 1506.

(29) Heath, P. J .; Schurr, J. M. Macrmolecules 1992, 25, 4149.

(30) Manning, G. S. J . Chem. Phys. 1969, 51, 924.
(31) Dautzenberg, H.; J aeger, W.; Kotz, J .; Philipp, B.; Seidel, Ch.; Stscherbina, D. Polyelectrolytes: Formation, Characterization and Application; Hanser Publishers: New York, 1994.

(32) Smisek, D. L.; Hoagland, D. A. Macromolecules 1989, 22, 2270.

(33) Hoagland, D. A.; Smisek, D. L.; Chen, D. Y. Electrophoresis 1996, 17, 1151.

(34) Gao, J. Y.; Dubin, P. L.; Sato, T.; Morishima, Y. J . Chromatogr. A 1997, 766, 233.

(35) Tanahatoe, J . J .; Kuil, M. E. J . Phys. Chem. B 1997, 101, 5905.

(36) Essafi, W.; Lafuma, F.; Williams, C. E. J . Phys. II 1995, 5 , 1269.

(37) Cai, C.-H.; Romano, V.; Dubin, P. L. J . Chromatogr. A 1995, 693, 251.

(38) Dubin, P. L.; Principi, J . M. Anal. Chem. 1989, 61, 780. MA010046S 